\title{
A New Numerical Method for Optimal Control of a Class of Hybrid Systems
}

\author{
Radiss Alipour* \\ Faculty of Technical and Engineering, Electronic Department, Shahid Bahonar, Kerman, Iran; \\ radiss.alipour@hotmail.com
}

\begin{abstract}
Optimal control of uncertain hybrid systems is a major concern in control engineering. For optimal control of hybrid systems, there are a variety of direct methods, including parametric control and state-based parametric control. A reason that indirect methods are less used for optimal control of hybrid systems is difficulty in working with it and its primary valuing. The present study develops a new numerical method for optimal control of hybrid systems which decreases special restrictions of optimal control functions of hybrid systems per provided solution by a Bellman inequality. The obtained results show that an optimal control problem can be easily solved by converting it to an optimization problem. In addition, the used method obtained more accurate numerical value of the performance index. The results showed that the proposed method leads to greater convergence of the algorithm used in optimal control problems. The efficiency and performance of the proposed method was tested by an application example.
\end{abstract}

Keywords: Bellman Inequality, Hybrid Systems, Optimal Control, Optimization Problems

\section{Introduction}

Optimal control of hybrid systems plays a key role in many applications, including economics, finance and engineering. The purpose of this category of controls is to minimize cost or maximize revenue. This goal can be achieved by finding an optimal case for closed loop system or in some cases open-loop systems. Direct and indirect methods are two main ways to resolve optimal control problems. In indirect method, the problem changes to the other problem before solving and it is classified into Bellman planning and Pontryagin maximizing principle ${ }^{1-4}$.

Hybrid systems include systems which are the result of interactions between discrete and continuous dynamical systems. The study of such systems has been further accelerated in recent years. In most cases, these systems can be described and modelled by a discrete and continuous system in a certain framework considering its primary objective $^{5-7}$.

One of the main reasons of recent interest in these systems is a combination of different mathematical models and their simple simulation. Control systems generally depend on their operating range of application, including switching between multiple modes. In recent years, it has been tried to study and review various aspects of hybrid systems ${ }^{8}$.

The system stability has been one of the hottest topics in applied research in the last decade. Stability and optimal control of hybrid systems are not exempt from this rule and great deal of research has been done on it. A number of studies examined the stability of discrete systems by relevant methods and others examined continuous systems for hybrid systems ${ }^{9}$. Single-stage and multi-stage hybrid systems are important for optimal control. The two systems differ in the amount and complexity of the application ${ }^{10,11}$.

The present study develops a new numerical method for optimal control of a class of hybrid systems by dynamic programming and optimizing properties. Bellman's inequality used in this study leads to decreased restrictions special for optimal control functions of hybrid systems per developed solutions and optimal costs under 
linear planning conditions. Considering the studied problem and uncertainties of multi-stage hybrid systems, the present study will use single-stage systems to develop a new numerical method for optimal control of hybrid systems.

Stochastic Hybrid Systems are an important class of hybrid systems including models in which the behavioural variations around the impulse response are related to their stochastically systemic behaviour. For analysis of these systems, which are commonly observed in response to unwanted mutations, general approximates of Markov decision processes are used. The selection process is based on a mathematical model to model decision-making function in situations where the outputs are random but somewhat under control; i.e. they have variations which are not controllable around a specific function and they can be more effective by a dynamic programming. In addition, the desired approximation can be achieved by Markov function discretization, especially for systems which are not directly observable or controllable, if needed. Using this approximation, middle and the covariance are preserved; this causes that the considered approximation does not trigger error, except in the acceptable range. To approximate boundary conditions of these systems, their dynamic mode around the minimum and maximum limits is approximated by random changes. To determine the general behaviour and stability of these systems, conventional method of discretization, which is the basic method for solving optimal control problems, is used. It is noteworthy that these approximations and thus acceptability of the errors triggered while random discretization and approximation are determined by conditions of the system such as the required accuracy and sensitivity of the examined unit ${ }^{12,13}$.

Production and reproduction hybrid systems take advantage of optimal control. In modeling and planning of production units, cost and profit can be approached to optimal by dynamic planning. Considering the fact that production cost varies in high and low quality units, optimal can be achieved using optimal control by extracting cost function related to the process and independent examination of the cost and profit ${ }^{14}$.

In systems which are conditionally stable and may be unstable for some initial conditions or inputs, system can be stable for all scenarios using a feedback. If the system is time-invariant, the key problem is to determine the terms and amounts required for system stability; in most cases, this can be achieved only by approximations. Therefore, the optimal boundary conditions can be achieved by optimal control and the best conditions ${ }^{15}$. In problems of non-linear systems, for example, the system is linearized by controlling variables and possible paths. Due to the fact that the system is not ideal and particularly its non-linear relations, divergence can be avoided by approximations derived from optimal control methods and using polynomials ${ }^{16}$.

\subsection{Framework of Single-stage Hybrid Systems}

Figure 1 shows a single-stage hybrid system. A sequence of $\mathrm{N}$ successive operations allocated from an external source are inserted for processing in defined times $0 \leq \mathrm{a}_{1}$ $\leq \ldots \leq \mathrm{a}_{\mathrm{n}}$. At this stage, the various activities which need to be processed by the system are denoted by $\mathrm{Ci}, \mathrm{i}=1, \ldots \mathrm{N}$.

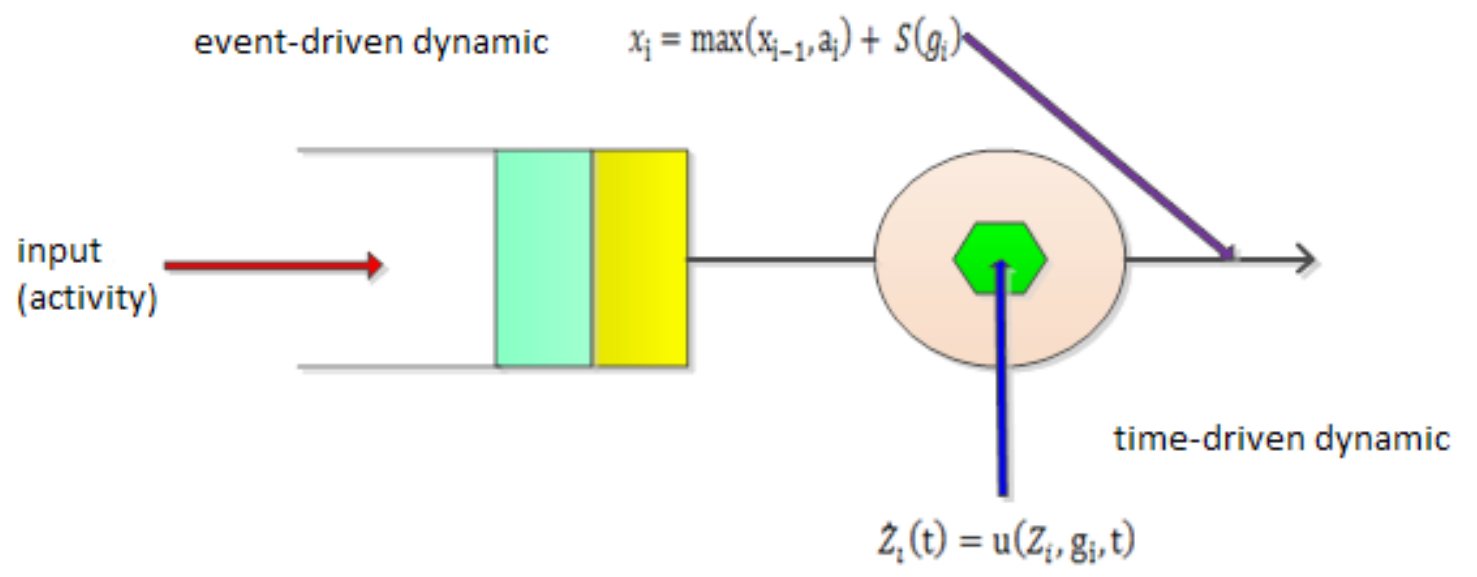

Figure 1. Representation of a single-stage hybrid system ${ }^{5}$. 
Activities are processed as the "FIRST COME-FIRST SERVED" (FCFS). Processing time is denoted by $S\left(g_{i}\right)$ as a function of the control variable $u_{i}$. In general, control of different times of processing is a function of processing time $\mathrm{S}_{i}$. For limited constant controls during a certain activity, it is enough to evaluate that processing times $S_{i}$ are not negative ${ }^{17-19}$. For example: $S\left(g_{i}\right) \geq 0$.

Time-driven dynamic: an activity $\mathrm{Ci}$ in time $\mathrm{x} 0$ in a physical state $\mathrm{Ni}$ is defined as a time-driven dynamic, according to (1):

$$
\dot{Z}_{i}(\mathrm{t})=\mathrm{u}\left(Z_{i}, \mathrm{~g}_{\mathrm{i}}, \mathrm{t}\right), \quad Z_{\mathrm{i}}\left(\mathrm{x}_{0}\right)=\kappa_{\mathrm{i}}
$$

Event-driven dynamic: completion time of an activity is denoted by $\mathrm{x}_{\mathrm{i}}$. In addition, it will be formulated by the following standard relation.

$$
x_{\mathrm{i}}=\max \left(\mathrm{x}_{\mathrm{i}-1}, \mathrm{a}_{\mathrm{i}}\right)+S\left(g_{i}\right), i=1, \ldots N
$$

It should be noted that the choice of control variable $g_{i}$ to adjust normal and uniform hybrid system is influenced the physical state $Z_{i}$ and temporal state $x_{i}$. For the singlestage hybrid system defined by (1) and (2), the purpose of optimal control is to select a control sequence $\left\{\mathrm{g}_{1}, \ldots, \mathrm{g}_{\mathrm{N}}\right\}$ to minimize the objective function $\mathrm{J}$ as defined below.

$$
J=\sum_{i=1}^{N}\left\{\theta\left(g_{i}\right)+\varphi\left(x_{i}\right)\right\}
$$

\section{Formulation}

Set (4) is considered as a hybrid system; so that, $x(t) \in X \subset R^{n}$ includes the state vector, and $u(t) \in \Omega_{u} \subset R^{m}$ contains a continuous input signal.

$$
\left\{\begin{array}{l}
\dot{x}(t)=f_{q(t)}(x(t), u(t)) \\
q(t)=v\left(x(t), q\left(t^{-}\right), \mu(t)\right)
\end{array}\right.
$$

In this system, $\mu(t) \in \Omega_{\mu}$ is discussed as a discrete input which allows the system to select different modes $q(t) \in Q=\{1,2, \ldots, N\}$. In this set, $q\left(t^{-}\right)$represents symmetric $q(t)$ in the time axis. With regard to relationships, only when the condition shown in Equation (5) is satisfied, the state $q$ can be shifted to state $r$. It should be noted that time argument is eliminated from the sequence in order to increase readability and clarity.

$$
x \in S_{q, r} \subseteq X
$$

The main problem of optimal control is minimization of the cost function, as shown in (6).

$$
J\left(x_{0}, q_{0}\right)=\int_{t_{0}}^{t_{f}} l_{q}(x, u) d t+\sum_{k=1}^{M} s\left(x\left(t_{k}\right), q\left(t_{k}^{-}\right), q\left(t_{k}^{+}\right)\right)
$$

Our problem is to shift a system from an initial state $\left(x_{0}, q_{0}\right)$ in time $t_{0}$ to a final state $\left(x_{f}, q_{f}\right)$ in time $t_{f}$ in which $\mathrm{M}$ is the number of steps for shifting a system from an initial state to a final state under the considered conditions, as shown in (7):

$$
t_{0}<t_{1}<t_{2}<\ldots<t_{M}<t_{f}
$$

where, $s(q, r, t)>0$ is the corresponding cost for shifting system from discrete state $\mathrm{q}$ to state $\mathrm{r}$. It is noteworthy that, $s(\cdot)>0$ leads to elimination of infinite steps for shifting from a discrete state $\mathrm{q}$ to a discrete state $r$ in a fully certain and assured form.

The framework developed in this paper changes the number of continuous states based on discrete states according to $\dot{x}_{q}(t)=f_{q(t)}\left(x_{q}(t), u_{q}(t)\right)$ in which $x_{q}(t) \in X_{q} \subset R^{n(q)}, u_{q}(t) \in \Omega_{u_{q}} \subset R^{m(q)}$.

\subsection{Constraints Required for Cost Optimization}

To avoid divergence of the final solution, it is necessary to set out the basic constraints to be imposed on the cost function. These constraints cause limitations on the path to reach optimal solutions of the cost function. Thus, the following conditions can be applied on the functions and inputs used in the optimization problem.

First condition: Continuous input function $u(t)$ is strictly ascending/descending, continuous and differentiable; for all values, we have (8):

$$
|u(t)|<\infty
$$

Second condition: Discrete input function $\mu(t)$ is strictly ascending/descending, continuous and differentiable; its minimum value is obtained at a finite value.

Third condition: For cost functions and control functions, (9) is true:

$$
0 \leq \frac{\partial V_{q}(x)}{\partial x} f_{q}(x, u)+l_{q}(x, u)
$$

Three conditions stated above are requirements to meet basic physical needs of hybrid systems. In addition 
to the conditions listed above, there are a number of remarks in order to achieve the most optimal scenario:

Remark 1: With regard to the relations of (10) and (11):

$$
\begin{gathered}
x_{j k}=x_{f}+j h e_{1} k h e_{2} \\
x^{j k}=\left\{x_{j k}+\alpha_{1} h e_{1}+\alpha_{2} h e_{2}: 0 \leq \alpha_{i} \leq 1\right\} \\
\hat{X}^{j k}=\left\{x_{j k}+\alpha_{1} h e_{1}+\alpha_{2} h e_{2}:-1 \leq \alpha_{i} \leq 1\right\} \\
\left(f_{-q}^{j k}\right)_{i}=\min _{x \in \bar{X}^{j k}, u \in \Omega_{u}}\left(f_{q}(x, u)\right)_{i} \\
\left(f_{q}^{-j k}\right)_{i}=\max _{x \in \bar{X}^{j k}, u \in \Omega_{u}}\left(f_{q}(x, u)\right)_{i} \\
\left(l_{-q}^{j k}\right)_{i}=\min _{x \in \bar{X}^{j k}, u \in \Omega_{u}}\left(l_{q}(x, u)\right)_{i}
\end{gathered}
$$

Assuming that the problem addressed in this paper can be developed, (11) there will be no change considering $\mathrm{N}$ shift instead of $\mathrm{M}$ shifts during $\left(t_{0}, t_{f}\right)$ in which $\mathrm{N}=\mathrm{M}+\mathrm{d}$ as well as $\mathrm{d}$ which is a positive integer, except the change in the number of states from $\mathrm{N}$ to $\mathrm{M}$.

Remark 2: in some problems, some subsystems are required to be added to the main body of the problem to reduce the complexity of the system. In this case, outputs related to this subsystem can be calculated independently, without consideration of the main body by initial conditions. As a result, the proposed method can be easily extended to any finite number of shifts (AND/OR).

Remark 3: in some problems, the number of restrictions of the solutions decreases due to the initial conditions of the problem. This reduces the time required for calculation of the optimal solution and thus cost function. Therefore, the minimum value can be easily obtained.

\section{A Practical Example}

To present the performance of the method suggested for optimal control of hybrid systems, a practical example is developed and simulated.

Example 1: Let us consider the following system

$$
\left\{\begin{array}{c}
x^{\prime}=z \\
z^{\prime}=L_{k}(z) u ; k=1,2 ;|u| \leq 1
\end{array}\right.
$$

where, $L_{k}(z)$ is represented in Figure 2. This system indicates a factory with three different production lines which are totally programmed for a similar activity and act in a parallel form.

As shown in Figure 2, values of these functions at various activity periods experience different changes. However, function inputs are the same homogenous except for the initial and end points of activity points. For

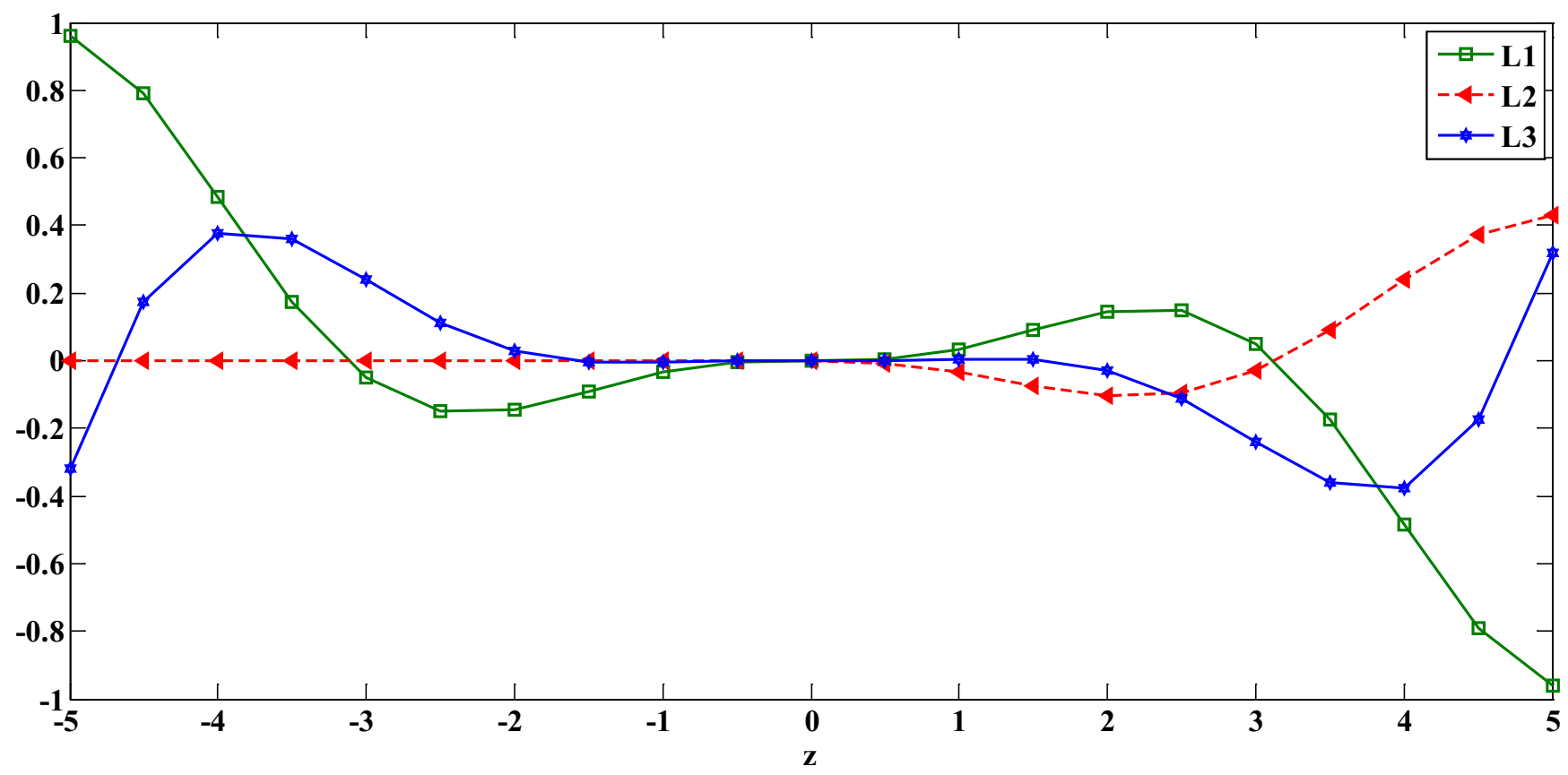

Figure 2. Representation of inputs to hybrid systems. 
optimality and optimal control of hybrid systems, values of input functions need to be similar. This similarity results from optimal control of hybrid systems. Figure 3 shows optimality and maximized performance of hybrid in heterogeneous activity periods.

As Figures 3 and 4 show, optimal control of inputs of a hybrid heterogeneous system can provide a more accurate numerical value to reach maximum yield and minimum cost resulting from homogeneous and heterogeneous activities of a hybrid system. Figure 3 shows that optimal control of inputs considerably increases optimality and performance of hybrid systems in heterogeneous activity periods. This will reduce cost of parallel activities.

\section{Discussion}

Optimal control of hybrid-dynamic systems is a relatively new discussion which determines inputs of a dynamic system for optimization (minimization and maximization) of performance specified in a system to meet requirements and limitations imposed on the studied system. Because of many complex applications of hybrid systems, optimal control problems are often formulated and solved by numerical methods. Numerical methods to solve optimization problems dates to Bellman's work, since application of numerical discussions in solving optimal control, many complexities have been triggered in optimal control problems and their applications; in addition, numerical solutions of these problems have considerably changes, which both add to accuracy of solutions and extend their application in different problems.

Numerical methods for solving optimal control problems can be divided into two main classes: 1) direct methods, 2) indirect methods. A direct numerical method calculates the main variables to determine the first order optimality conditions of the original optimal control problem. The indirect method solves multiple and local boundary conditions to determine the most optimal path to solve the optimal control problem. Then, it is discussed that whether the determined path leads to minimization/ maximization of the optimal control problem.

To evaluate the efficiency of production units using multidimensional dynamic programming and Bellman inequality, the present study tried to solve an optimal control problem for a class of hybrid systems. Observations obtained from these inequalities in the numerical solution indicate its good results compared with other numerical methods. The present study also showed that direct and indirect numerical hybrid methods lead to more accurate results than using only one method.

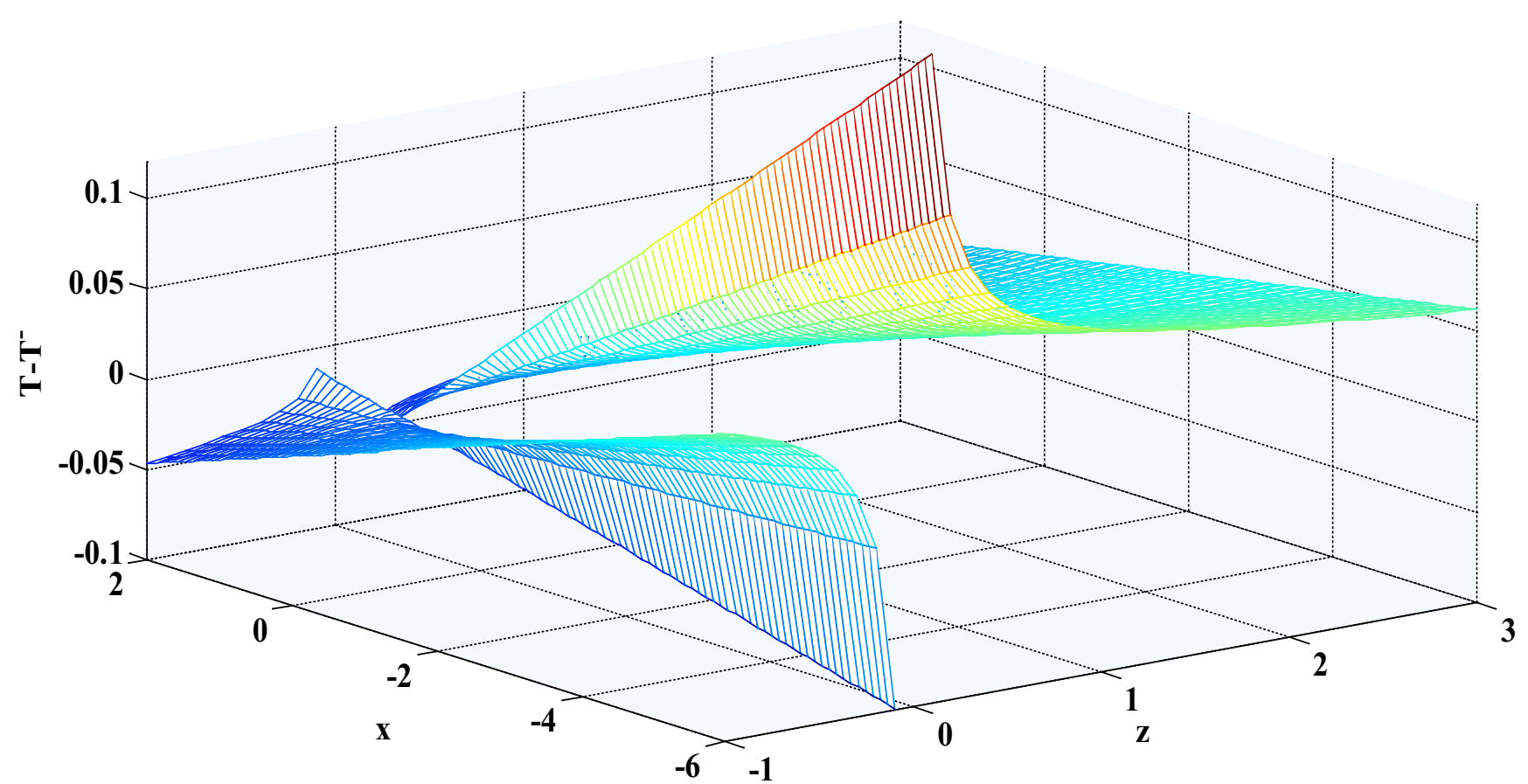

Figure 3. Representation of optimality and maximized performance of the hybrid systems in heterogeneous activity periods. 


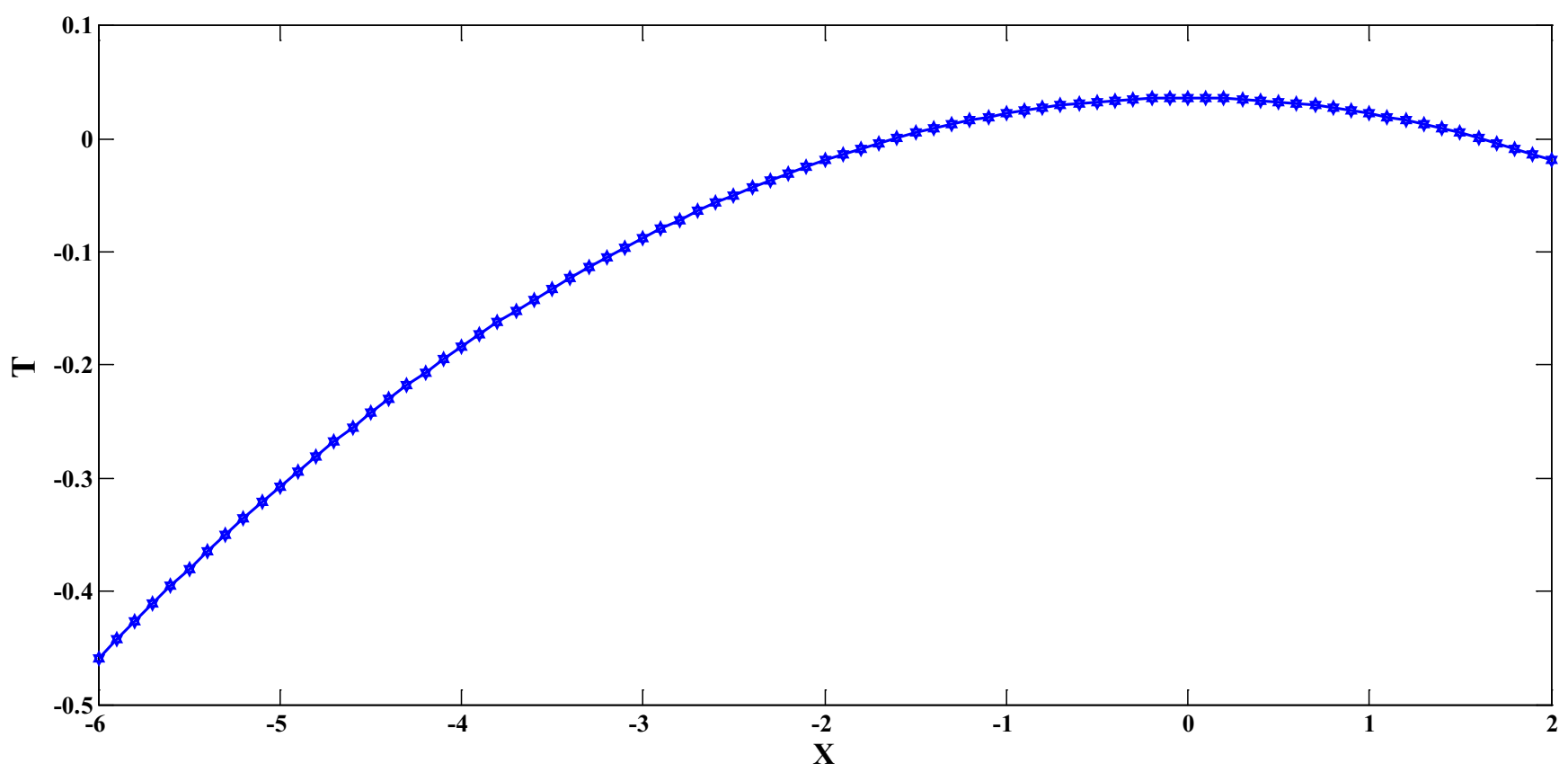

Figure 4. Numerical value of the hybrid system performance.

\section{Conclusion}

Hybrid systems are a combination of discrete and continuous systems. The present study used a developed model of Bellman inequality to calculate optimal cost function and its application in a linear planning and discretization. Calculations show that the techniques used for optimal control need to be both consistent with theoretic optimal control and satisfying the calculating conditions. The results showed that an optimal control problem can be solved easily by changing it into an optimization problem. The present study focused on the continuous part of a hybrid system and avoided accurate examination of the discrete part which itself consists of several cases. The applied method obtained more precise numerical value of the performance index. The results showed that the proposed method leads to greater convergence of the algorithm used in optimal control problems.

Considering the used example, when values of the input functions to hybrid systems are in one range (with and without normalization), the considered algorithm leads to the best (optimal) case. Variations of the input functions and as a result their corresponding outputs show that output will be symmetric in cases in which the input is relatively symmetric in its relevant range. When this symmetry is closer to ideal, the obtained results will approach to the optimal case. The diagram of numerical value of the performance index in hybrid systems shows that its variations is less in points related to the end values where the diagram is flatter; this indicates system stability per value.

\section{References}

1. Hedlund S, Rantzer A. Optimal control of hybrid systems, Proceedings of the 38th IEEE Conference on Decision and Control, 1999; IEEE; 1999. p. 3972-7.

2. Branicky MS, Borkar VS, Mitter SK. A unified framework for hybrid control: model and optimal control theory. IEEE Transactions on Automatic Control. 1998; 43(1):31-45.

3. Borrelli F. Constrained optimal control of linear and hybrid systems. Lect Notes Contr Inform Sci. 2003; 290.

4. Pepyne DL, Cassandras CG. Optimal control of hybrid systems in manufacturing. Proceedings of the IEEE. 2000; 88(7):1108-23.

5. Cho YC, Cassandras CG, Pepyne DL. Forward algorithms for optimal control of a class of hybrid systems. Proceedings of the 39th IEEE Conference on Decision and Control, 2000; IEEE; 2000. p. 975-80.

6. Shaikh MS, Caines PE. On the optimal control of hybrid systems: Optimization of trajectories, switching times, and location schedules. Hybrid systems: Computation and Control. 2003; 2623:466-81.

7. Cassandras CG, Pepyne DL, Wardi Y. Optimal control of a class of hybrid systems. IEEE Trans Automat Contr. 2001; 46(3):398-415. 
8. Sussmann HJ. A maximum principle for hybrid optimal control problems. Proceedings of the 38th IEEE Conference on Decis Contr. 1999; IEEE; 1999. p. 425-30.

9. Sciarretta A, Back M, Guzzella L. Optimal control of parallel hybrid electric vehicles. IEEE Trans Contr Syst Tech. 2004; 12(3):352-63.

10. Borrelli F, Baotic M, Bemporad A, Morari M. Dynamic programming for constrained optimal control of discrete-time linear hybrid systems. Automatica. 2005; 41(10):1709-21.

11. Shaikh MS, Caines PE. On the hybrid optimal control problem: theory and algorithms. IEEE Trans Automat Contr. 2007; 52(9):1587-603.

12. Temoçin BZ, Weber GW. Optimal control of stochastic hybrid system with jumps: A numerical approximation. J Comput Appl Math. 2014; 259: 443-51.

13. Smallwood RD, Sondik EJ. The optimal control of partially observable Markov processes over a finite horizon. Operations Research. 1973; 21(5):1071-88.
14. Cai X, Lai M, Li X, Li Y, Wu X. Optimal acquisition and production policy in a hybrid manufacturing/remanufacturing system with core acquisition at different quality levels. Eur J Oper Res. 2014; 233(2):374-82.

15. Corona D, Giua A, Seatzu C. Stabilization of switched systems via optimal control. Nonlinear Analysis: Hybrid Systems. 2014; 11:1-10.

16. Marzban HR, Hoseini SM. A hybrid approximation scheme for discretizing constrained quadratic optimal control problems. J Franklin Inst. 2014 May; 351(5):2640-56.

17. Shaikh M S. Optimal control of hybrid systems: Theory and algorithms [PhD thesis]. McGill University; 2004.

18. Bengea SC, DeCarlo RA. Optimal control of switching systems. Automatica. 2005; 41(1):11-27.

19. Delprat S, Lauber J, Guerra TM, Rimaux J. Control of a parallel hybrid power train: optimal control. IEEE Trans Veh Tech. 2004; 53(3):872-81. 